\title{
Harmonic Suppression In Microstrip Dual-Mode Ring-Resonator Bandpass Filters
}

\author{
U. Karacaoglu †, D. Sanchez-Hernandez $\dagger$, I. D. Robertson $\dagger$, and M. Guglielmi $\dagger \dagger$ \\ $\dagger$ Communications Research Group \\ Dept. of Electronic Engineering \\ King's College London \\ Strand, London, UK, WC2R 2LS \\ †† European Space Research \\ and Technology Centre (ESTEC) \\ Nordwjik. \\ The Netherlands
}

\begin{abstract}
This paper introduces two new topologies for rejecting the harmonics in microstrip ring resonator filters. First one uses $50 \Omega$ spur line filter structures placed at the input and output of the ring to suppress the first harmonic. Second topology incorporates low-pass structures into the ring for elimination of the harmonics.
\end{abstract}

\section{Introduction}

$\mathrm{Wu}$ and Rosenbaum [1] indicated the possibility that the TM110 resonance, using their mode definitions, could be excited twice on the same physical ring thereby creating a dual-mode microstrip ring resonator. This feature of the microstrip ring is similar to the dual-mode resonator in cylindrical waveguide, which is extensively used in filter design. Wolff [2] presented experimental evidence of the excitation of the degenerate modes. The realisation of microwave filters using dual-mode ring resonators was first presented by Guglielmi and Gatti [3] at the 1990 European Microwave Conference. The technique is particularly attractive because of its ability to implement two transmission poles and two transmission zeroes with only one ring if a 'symmetric' coupling structure is employed. The technique is especially suitable for realising small lightweight filters for space applications. The possibility of enhancing this technique by using simple geometry to make the circuit less sensitive to fabrication tolerances and easier to simulate acurately has been demonstrated by [4]. This 5.4 $\mathrm{GHz} 2$-pole planar filter, measuring only $15 \times 15 \mathrm{~mm}$, had a bandwidth of $300 \mathrm{MHz}$, with $1 \mathrm{~dB}$ insertion loss and high selectivity. However, due to the higher order resonances of transmission-line ring resonators the out-of-band rejection of the filter needs to be improved. This paper introduces two new topologies for rejecting this higher order resonances without disturbing the passband specifications.

\section{The Spur-line Band-stop Filter}

Bates [5] designed a microstrip spur-line band-stop fillter suitable for bandwidth of the order of $10 \%$ within the width of a microstrip transmission line as shown in figure 1 . The length of the spur a and the gap b determine the centre frequency fo given by:

$a=\frac{2.99792 .510^{8}}{4 f_{o} \sqrt{K_{\text {effo }}}}-\Delta l_{l}$

Where Keffo and $\Delta \mathrm{ll}$ are the odd-mode effective dielectric constant and the effective length extension due to the gap $b$ respectively. This filter forms a compact structure that is also virtually nondispersive. The behaviour of this filter can be represented by mears of an impedance matrix, derived by Jones and Bolljahn [6]. In Bates' analysis, which includes the effect of the capacitance between the end of the spur and the main line, the energy stored by the resonant structure was found to be mainly determined by the odd mode of propagation. It is well known that the microstrip propagation is not TEM and therefore the phase velocities for the odd and even mode are not equal; but assuming equal phase velocities the spur-line filter can be represented as the equivalent circuit given by Bates [5]. The band-stop spurline filter was designed to operate at the first harmonic frequency of the ring filter. Taking advantage of its inherent space-saving nature, the spur-line filter was then embedded in both the input and output $50 \Omega$ lines of the ring filter as shown in figure 2 . When cascaded, the resulting topology produced a significant suppression of the first harmonic, where a comparison of the simulated transmission and return loss of the ring filter with and without the spur-line filter is shown in figure 3 . Figure 4 shows the measured response of the spur line ring resonator filter it has $400 \mathrm{MHz}$ $3 \mathrm{~dB}$ bandwidth centered at $5.5 \mathrm{GHz}$ and the second 
harmonic is suppressed successfully. Figure 5 shows the photograph of the spurline filter.

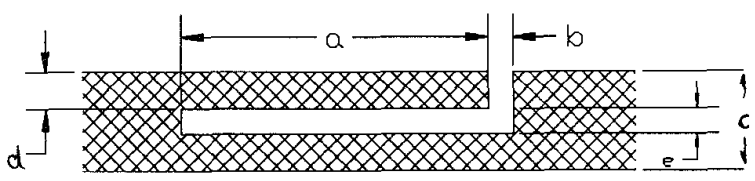

Fig. 1. Spur-line band-stop filter.

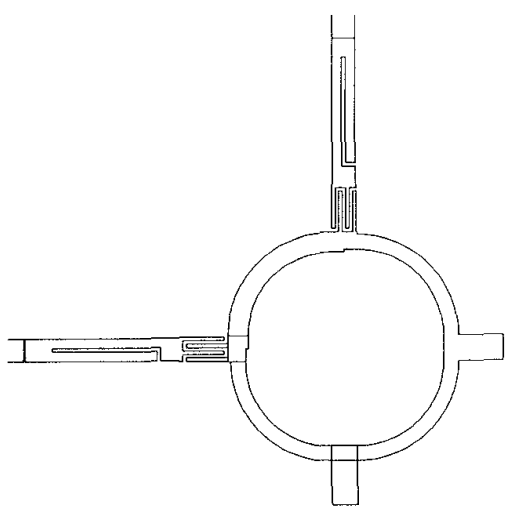

Fig. 2 Layout of the spur line ring resonator filter

\section{Low-Pass Ring Structures}

There are a three main ways in which a low-pass structure can be integrated into the rings for elimination of the unwanted higher frequency transmission bands:

1) using alternate sections of narrow and wide lines as shown in Fig. 6(a).

2) placing open circuit stubs on the ring to realise shunt capacitances Fig. 6(b)

3) placing radial stubs on the ring as shown in Fig. 6(c)

This structure can be termed a 'daisy' ring. With careful design a dual-mode ring without higher order resonances results. These behave as slow-wave structures and this leads to a further advantage which is that the size of the ring is reduced.

In principle, any of these three methods should work. However, the dual-mode ring is very sensitive to the symmetry and mode coupling discontinuities. Experimentally it has been found that method (1) gives the closest response to the normal ring.

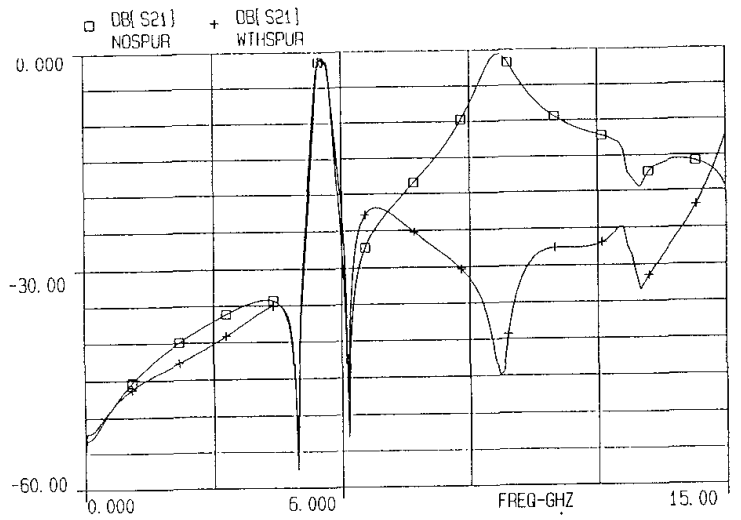

Fig. 3 Simulated response of the ring resonator filter with and without the spur line at the input and the output

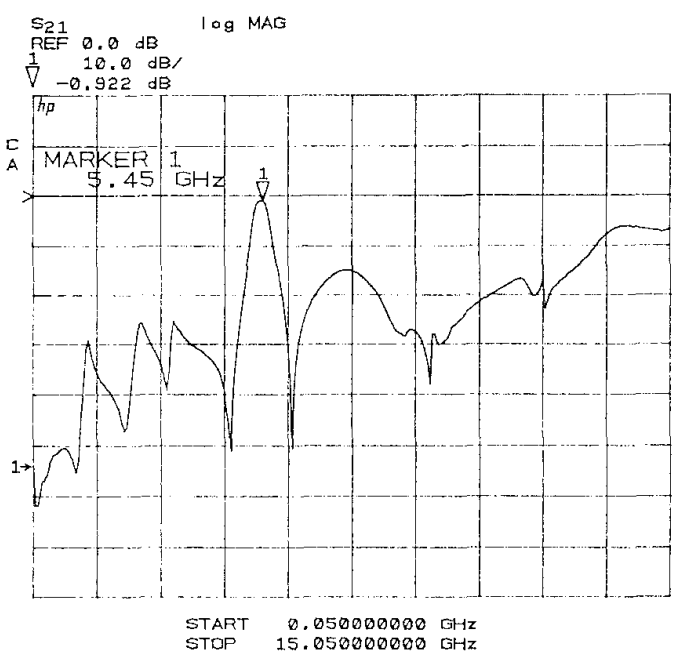

Fig. 4(a) Measured insertion loss of the spur line ring filter

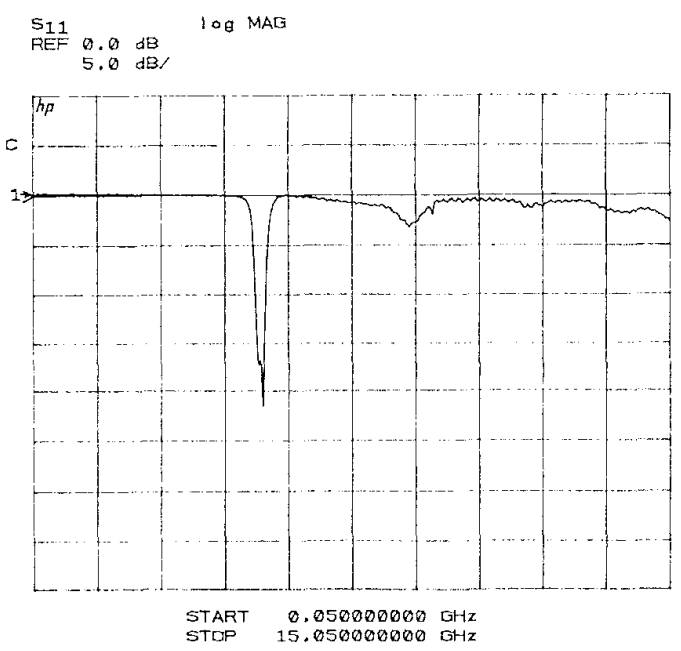

Fig. 4(b) Measured return loss of the spur line ring filter 


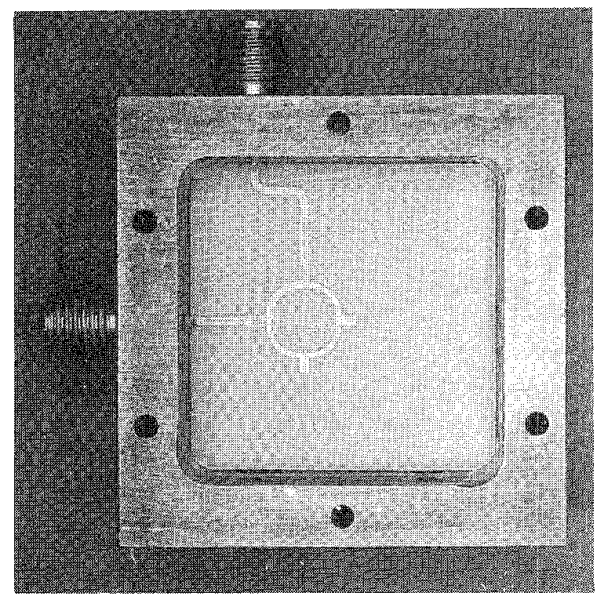

Fig. 5 Photograph of the spur line ring resonator filter

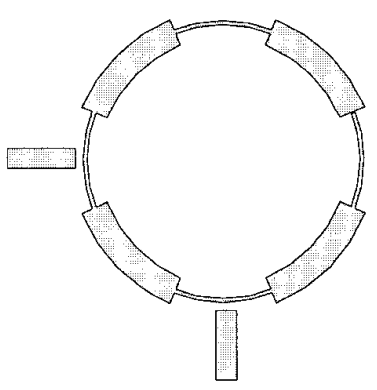

Fig. 6(a) Low-Z/ High-Z low-pass ring

Fig. 6(b) Low-pass ring with open circuit stubs
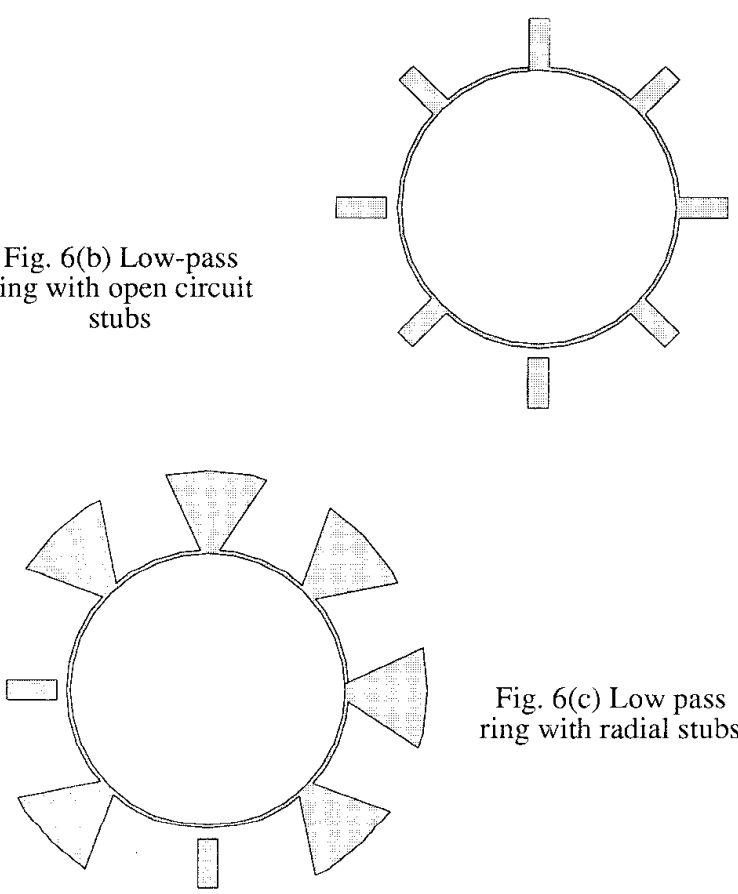

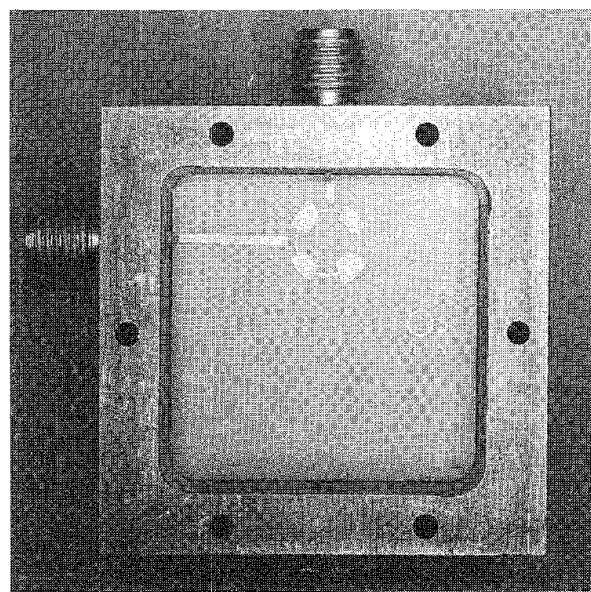

Fig. 7 Photograph of the dual-mode daisy ring filter

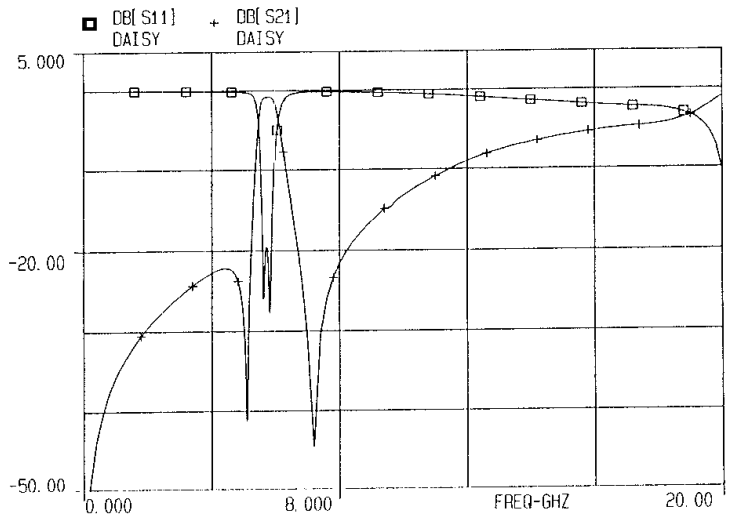

Fig. 8 Simulated response of the daisy ring filter

\section{Measurements}

A photograph of the finished dual-mode daisy ring filter is shown in Fig. 7. The pre-fabrication simulated response is shown in Fig. 8. The measured results of the filter are shown in Fig. 9. Absolutely no tuning was employed, and yet the agreement with predictions is excellent. The transmission response shows an excellent shape with a $500 \mathrm{MHz} 3-\mathrm{dB}$ bandwidth. Steep skirts are provided by the transmission zeros, and there are no spurious transmission bands from higher order resonances. The return loss response shows the positions of the two poles clearly. For comparison, Fig. 10 shows the measured response of a $5.25 \mathrm{GHz}$ dual-mode ring filter which did not use the low-pass structure: The problem of the higher order resonances is very clear. 


\section{Conclusions}

New techniques has been demonstrated for improving the out-of-band rejection of dual-mode microstrip ring resonator filters. A "daisy ring" $5.25 \mathrm{GHz}$ filter prototype has been designed and constructed and gives a nearly ideal response. The measured results show a $3-\mathrm{dB}$ bandwidth of $500 \mathrm{MHz}$ centred on $5.25 \mathrm{GHz}$. The filter has steep skirts as a result of the transmission zeros given by the dual-mode ring and has greatly improved high frequency rejection compared with the standard dual-mode ring. However in the small space of the ring it is difficult to realise a low-pass filter structure with a sufficiently high order to filter the second resonance fully. the alternative method is to remove second resonance with a band-stop filter element. This has been successfully demonstrated by using a spur-line bandstop filter within the feedlines of the ring. This attenuates the second resonance by over $30 \mathrm{~dB}$, with no size penalty.

\section{References}

[1] Y.S. Wu and F.J. Rosenbaum, "Mode chart for microstrip Ring Resonators", IEEE Trans. Microwave Theory Tech ., July 1973, pp.487-489.

[2] I. Wolff, "Microwave Bandpass Filter Using Degenerate Modes of a Microstrip Ring Resonators ", Electron. Lett., 1972, pp.163-164.

[3] M. Guglielmi and G. Gatti, "Experimental Investigation of Dual-Mode Microstrip Ring Resonators", Proceedings of the 20th European Microwave Conference, Budapest, Sept 1990, pp.901-906.

[4] U. Karacaoglu, I. D. Robertson, and M. Guglielmi, " An Improved Dual-Mode Microstrip Ring Resonator Filter with Simple Geometry ", Proceedings of the 24th European Microwave Conference, Cannes, Sept. 1994.

[5] Bates, R.N.,'Design of microstrip spur-line band-stop filters,' IEE Int. Journal on Microwaves, Optics and Acoustics, Nov, 1977, vol. 1, no. 6, pp. 209-214.

[6] Jones, E.M.T. and Bolljahn, J.T.,'Coupled-striptransmission lines filters and directional couplers', IRE Trans. on Microwave Theory \& Tech., 1956, 5, pp. 7

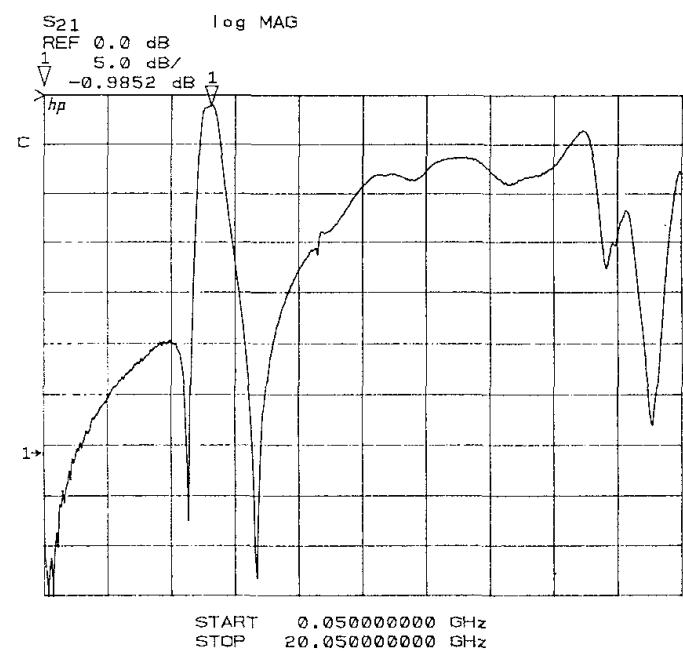

Fig. 9(a) Measured insertion loss of daisy ring filter S11
REF $0.0 \mathrm{~dB}$
$4.0 \mathrm{~dB} /$

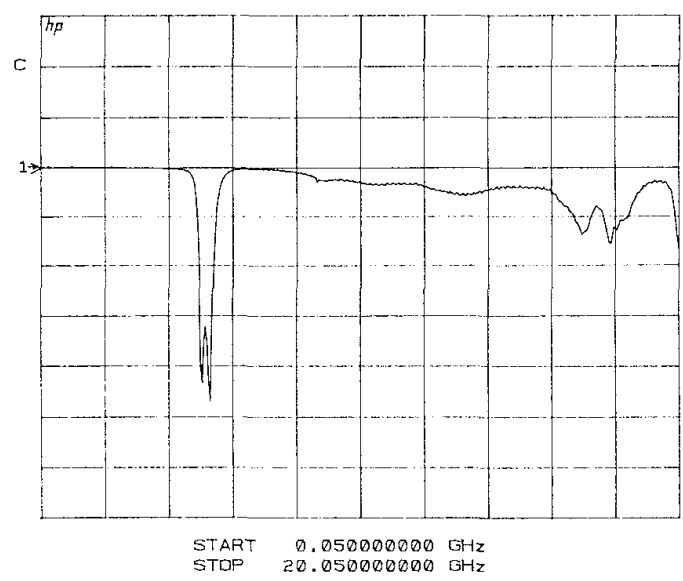

Fig. 9(b) Measured return loss of daisy ring filter 521.0 log MAG

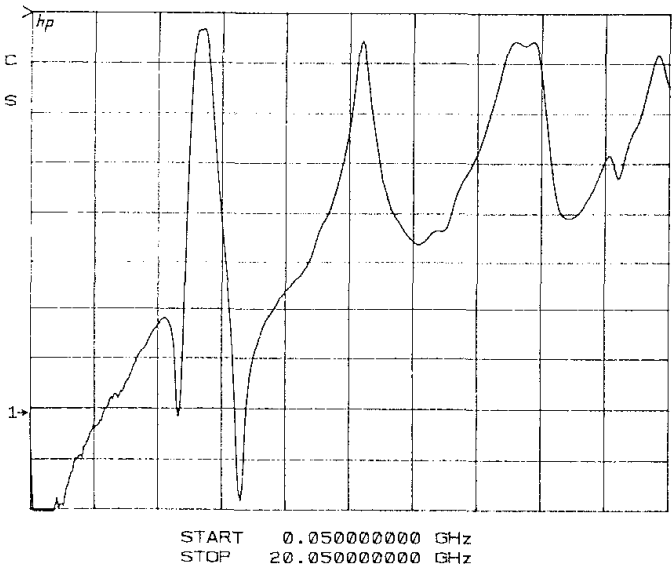

Fig. 10 Measured insertion loss of standard dual-mode ring 